\title{
Die Wortstellung des Subjekts „Gott“ im Koran
}

\author{
YEHUDIT DROR (Haifa)
}

\begin{abstract}
In diesem Aufsatz wird ein Aspekt der Frage Wortstellung im Koran behandelt und zwar die Stellung des Subjekts 'allāh und rabb in den einfachen Verbalsätzen im Koran. ${ }^{1}$ Um die bestehenden Wortstellungstypen darzustellen, werden die Sätze in drei Gruppen klassifiziert: Klassifikation nach Verb- und Aktionsart, Klassifikation nach der Satzart und Klassifikation nach Satzeinleitungspartikeln. Insgesamt konnten vier Ursachen erarbeitet werden, die zu den verschiedenen Wortstellungen führen: Tempus und Aspekt, unterschiedliche Funktionen der Partikeln und der Pronomina, sowie rhetorische Zwecke und Betonung.

This article examines one aspect of word order in the Qur'ann, which is the position of the subjects 'allah and $r a b b$ in simple verbal sentences. To present the word order types existing in the Qur'ān, the sentences were classified in three groups: the verb's type and its aspect (aktionsart), the sentence type, and the type of the particle preceding the sentence. Four factors affecting the word order were found: time and aspect, different functions of a specific particle, rhetorical purposes, and emphasis.
\end{abstract}

\section{Einleitung}

Beispiele für die Stellung des Subjekts „Gott“ im Koran finden sich in verschiedenen Werken, die jeweils eine Erklärung für diese Wortstellung liefern. Beispielsweise führt Suleiman (1989) Sure 39:42 an, wo das Subjekt „Gott“ am Satzanfang steht und dadurch betont wird, dass nur Gott in der Lage ist, die Menschen abzuberufen, wenn sie sterben:

The most obvious reason for subject preposing is emphasis [...] Let us consider the following examples from the Qur'ān:

$\begin{array}{llll}\mathrm{S} & \mathrm{V} & \mathrm{O} & \\ \text { 'allāhu } & \text { yatawaffā } & \text { l-'anfusa } & \text { hinna mawti-hā } \\ \text { God } & \text { take } & \text { souls } & \text { on death their (GEN) }\end{array}$

"God takes souls in the moment of death" which shows that preposing the subject 'allahu' 'God' is meant to assert that the act of taking one's soul after death is no one's business but God's. ${ }^{2}$

1 Die Ergebnisse gründen sich auf meine Doktorarbeit, in der die Wortstellung in den einfachen Verbalsätzen im Qur'ān behandelt wird.

2 Suleiman 1989: 222.

Journal of Arabic and Islamic Studies • 14 (2014): 63-86 (c) Yehudit Dror, University of Haifa, Department of Arabic Language and Literature, Israel 
Suyūṭ̂̄ behandelt in seinem Werk al-'Itqān fì 'ulūm al-Qur'ān die Frage der Wortfolge. Dabei erwähnt er auch die Voranstelllung des Subjekts „Gott“. Der Grund für diesen Wortstellungstyp lautet bei ihm: ta'żim ,Ehrenbezeugung'. Durch die Voranstellung des Subjekts 'allāhu am Anfang wird ihm gegenüber Ehre bezeugt. ${ }^{3}$

Es ist vor allem der Artikel von Dahlgren (2001) zu erwähnen, der von der Wortstellung und Topikalität im Koran handelt. Im Zentrum dieses Artikels steht die Wortstellung des Subjekts „Gott“. Während der Arbeit an seiner Dissertation bemerkte Dahlgren, dass der Prozentsatz in Sätzen mit der Wortstellung SV und dem Subjekt „Gott“ sehr hoch ist. Seine Erkenntnisse in Bezug auf das Subjekt „Gott“ sind die folgenden:

1. In der Einleitung des Artikels behandelt er die Satzstruktur, die die Schule von Prag aufstellte. Laut dieser Schule besteht ein Satz aus „Rhema“ und „Thema“. Der letzte Begriff bezieht sich auf das, was bekannt bzw. klar ist oder eine Sache, über die man spricht. Rhema ist das, was über das Thema ausgesagt wird. Für die Kriterien, welche die Satzelemente erfüllen müssen, die das Thema sind, bezieht sich Dahlgren in seiner Analyse auf verschiedene Hierarchien aus Givóns Werken:

- existential $^{4}>$ indefinite $>$ definite $>$ anaphoric pronoun $^{5}$

- $\quad$ subject $>$ direct object $>$ others ${ }^{6}$

- definite > indefinite

- more involved participant ${ }^{7}>$ less involved participant

- $\quad$ speaker $\left(1^{\text {st }}\right.$ pers. $)>$ listener $\left(2^{\text {nd }}\right.$ pers. $)>$ other $\left(3^{\text {rd }}\right.$ pers. $)$

- human > non-human

Laut der sechs Kriterien der Hierarchien haben Subjekt, bestimmtes Substantiv, involvierte Teilnehmer an einem Ereignis, Sprecher in einer Erzählung und Personen bezeichnendes Subjekt (belebtes Subjekt) eine hohe Wahrscheinlichkeit, das Thema zu sein.

Angesichts seiner statistischen Ergebnisse ${ }^{8}$, bzw. der Sätze mit dem vorangestellten Subjekt „Gott“, fügt Dahlgren eine weitere Kategorie ein: superhuman > human > nonhuman.

3 SUYŪṬī 1951, I (an-naw' ar-rābi` wa-l-'arba'ūna fì muqaddama wa-mu'ahhara): 14. Diese Erklärung ist vergleichber mit der folgenden Erklärung für die Voranstellung des Subjekts „Gott“ im Alten Testament. MURAOKA 1985: 35 sagt dazu: "S [Subjekt]=God It is possible that in certain expressions with the divine name or a divine messenger as the subject, a kind of religious psychology in which God occupies the dominant place determines the arrangement of words giving S the initial positions."

4 DAHLGREN 2001: 22 erklärt diesen Begriff folgendermaßen: "Existential sentences present a new theme, through such expressions as there is, e.g. Once there was a king... or more prosaic, There is a fly in the soap. The corresponding construction in Early and Classical Arabic is kāna followed by an indefinite noun in the nominative, e.g. kāna rağulun There was a man."

5 GIVÓN 1977: 182.

6 GIVÓN 1984, II: 901.

7 CRYSTAL 2003: 337: Mit „Partizipient“ bezeichnet man v.a. in der Pragmatik die an einer linguistischen Interaktion teilnehmende Person. Die typische Rollenverteilung sieht einen Sprecher und Empfänger vor, allerdings können auch andere Rollen eingenommen werden, wie die des Partizipienten. Dieser ist weder Ziel noch Ursprung der Nachricht.

8 S. Tabelle 2 auf Seite 25 in seinem Artikel. 
Das Subjekt „Gott“ (=superhuman) hat wegen seine Allmacht und seines Einflusses auf das Leben der Menschen eine größere Wahrscheinlichkeit am Satzanfang zu erscheinen.

2. In erzählenden Abschnitten, in denen wiederum „Gott“ als Subjekt fungiert, herrscht die Wortstellung VS vor.

3. In Dialogen ist die übliche Wortstellung VS.

4. Die Wortstellung nach dem Demonstrativpronomen ka-dālika ist VS. Nur in einem Satz mit dem Subjekt „Gott““ (von 30 gesammelten Sätzen) ist die Wortstellung SV. Der Grund für die Wortstellung SV wird bei Dahlgren nicht erklärt.

5. Die überwiegende Wortstellung der negativen Partikel $l \bar{a}$ zusammen mit dem Subjekt „Gott“ ist SV. Einer der Hauptgründe für diese Wortstellung ist die herausragende Stellung Gottes und deren grammatikalische Realisierung durch Voranstellung. Dennoch gibt es auch kontextgebundene Unterschiede. In den 41 Fällen mit der Wortstellung SV und Gott als Subjekt erscheinen 34 am Ende des Verses, dagegen erscheint von den 11 Fällen mit VS Stellung mit demselben Subjekt nur ein einziger am Ende des Verses.

Nach der negativen Partikel $m \bar{a}$ ist dagegen die übliche Wortstellung VS. Unter 22 zusammengestellten verneinten Sätzen, deren Subjekt „Gott“ ist, fand Dahlgren nur einen SVSatz (Q 3:108). ${ }^{9}$

Die Untersuchung aller einfachen Verbalsätzen im Koran, deren Subjekt „Gott“ ist, brachte weitere Erkenntnisse in Bezug auf die Frage der Stellung dieses Subjekts zutage, die im nächsten Abschnitt dargestellt werden. Dahlgren untersuchte auch erweiterte Sätze, wobei ich dem gegenüber gewisse Vorbehalte habe. Der Grund dafür ist, dass die verschiedenen Konjunktionen eine bestimmte Wortstellung verlangen, wie z.B 'inna und 'anna, die automatisch die Wortstellung SV nach sich ziehen. Aus diesem Grund beruht die vorliegende Forschung nur auf einfachen Verbalsätzen. ${ }^{10}$

Um die Wortstellungstypen besser darzustellen, wurden sie nach den folgenden Kategorien klassifiziert:

1. Verbart bzw. semantische Klassifizierung, Tempus (Perfekt, Imperfekt) und Aktionsart (abgeschlossene oder durative Handlungen, generelles Präsens)

2. Satzart: Negativ-, Fragesätze und Sätze „mit zwei Gesichtern“

3. Einleitungspartikel und Pronomen

9 DAHLGREN 2001: 32-33.

10 Mit „Verbalsätzen“ sind alle Sätze gemeint, die ein Verb enthalten, gleich welche Stellung im Satz vorliegt. Dies steht im Gegensatz zu der Definition des Begriffes „Verbalsatz“ bei den Nationalgrammatikern, die besagt, dass ein Satz als Verbalsatz betrachtet wird, wenn das Verb an der Satzspitze steht. Falls das Verb diese Stelle nicht besetzt, wird der Satz als Nominalsatz klassifiziert. 


\section{Verbart, Tempus und Aktionsart}

\subsection{Verb im Perfekt, Wortstellung VS}

In den untersuchten Sätzen dieser Kategorie steht das Verb im Perfekt und bezeichnet eine einmalige und abgeschlossene Handlung.

\subsubsection{Das Verb ḍaraba (mațalan) „ein Gleichnis prägen“}

(1) daraba llāhu matalan 'abdan mamlūkan lā yaqdiru 'alā šay'in (Q 16:75)

„Gott prägte ein Gleichnis: Ein Knecht, der versklavt ist und Macht über nichts hat ${ }^{\text {"11 }}$

\subsubsection{Das Verb qāla}

(2) wa-qāla llāhu 'innī ma'akum (Q 5:12)

„Und Gott sprach: Siehe, ich will mit euch sein“"12

Das Verb qāla im Perfekt ist eines der Charakteristika des Dialogs im Koran. Der Dialog im Koran kann in fünf Typen eingeteilt werden: Dialog zwischen dem Propheten und der Nation, an die er gesandet wurde (z.B.: Q 11:23-35), Dialog zwischen dem Propheten und Gott (z.B.: Q 2:260), Dialog, der im Jenseits stattfindet (z.B.: Q74:40-47), Dialog, der eine Beratung über ein bestimmtes Thema darstellt (z.B.: Q 12:8-10), und Dialog, in dem nur eine Seite bzw. ein Sprecher erwähnt wird (z.B.: Q 2:34-49). ${ }^{13}$

\subsubsection{Die Verben ğa áa und halaqa}

(3) ğáala llāhu l-ka bata l-bayta l-ḩarāma qiyāman li-n-nāsi (Q 5:97)

„Gott machte die Kaaba, das heilige Haus, zu einem Standort für die Menschen“"14

(4) halaqa llāhu s-samāwāti wa-l-'arḍa bi-l-haqqi (Q 29:44, 45:22)

„Gott schuf, in Wahrheit, den Himmel und die Erde“"15

Diese Sätze beschreiben nicht die Geschichte der Erschaffung, sondern sie besagen, dass Gott Himmel und Erde schuf. Hinzu kommt, dass kein Zusammenhang zwischen diesen Versen und den früheren Versen besteht. Sure 5:94-97 beschreiben das, was zu jagen erlaubt und verboten ist. In Sure 29:40-43 geht es um das Gleichnis der Spinne, deren Haus das schwächste ist. Sure 45:21 behandelt die Frage, ob die Ungläubigen mit den Gläubigen vergleichbar sind.

\footnotetext{
11 BoBZIN 2010: 236.

12 Ebd.: 94.

13 MUSTANSIR, Mir, “Dialogues”. Encyclopedia of the Qur'ān, 1: 532; vgl. auch MUSTANSIR 2004: 201202.

14 BOBZIN 2010: 105.

15 Ebd.: 348.
}

$J A I S \cdot 14$ (2014): 63-86 


\subsubsection{Verschiedene Verben, die in erzählenden Abschnitten erscheinen}

\section{Beispiele:}

Sure 2: 243-253: Die Erzählung von David und Goliath: ${ }^{16}$

(5) fa-hazamūhum bi-'idni llāhi wa-qatala dāwūdu ğālūta wa-'̄àtāhu llāhu l-mulka wa-lhikmata wa-'allamahū mimmā yašāu (Q 2:251)

„Und sie besiegten sie - mit Erlaubnis Gottes. Und David tötete Goliath, und Gott gab ihm die Herrschaft und die Weisheit und lehrte ihn etwas von dem, was er wollte". ${ }^{17}$

Verben im Perfekt bezeichnen vollständige und einmalige Ereignisse oder Handlungen, die in der Vergangenheit liegen. Aus diesem Grund werden Verben im Perfekt benutzt, um chronologische und nacheinander stattfindende Ereignisse $\mathrm{zu}$ beschreiben. ${ }^{18}$ Zusätzlich gehören die erwähnten Sätze mit Verb im Perfekt zu der Vordergrundinformation der erzählenden Abschnitte. Hopper definiert diese beiden Begriffe folgendermaßen:

The difference between the sentences in the foreground (the "main line" events) and the ones in the background (the "shunted" events) has to do with sequentiality. The foreground events succeed one another in the narrative in the same order as their succession in the real world [...] The backgrounded events on the other hand, are not in sequence to the foregrounded events, but are concurrent with them. Because of this feature of simultaneity, backgrounded events usually amplify or comment on the events of the main narrative. ${ }^{19}$

\subsubsection{Weitere Verben im Perfekt:}

(6) wa-kallama llāhu mūsā taklìman (Q 4:164)

„Und Gott sprach mit Mose unmittelbar“‘20

\subsection{Verb im Perfekt, Wortstellung SV}

In 13 Sätzen, deren Subjekt „Gott“ ist, steht das Verb im Perfekt und ebenso wie die oben erwähnten Verben bezeichnen sie eine einmalige und abgeschlossene Handlung. Aber in den folgenden Sätzen ist die Wortstellung SV, das Subjekt steht also in Anfangsstellung:

(7) wa-llāhu 'anzala mina s-samāi māàn (Q 16:65)

„Gott sandte aus dem Himmel Wasser hernieder“621

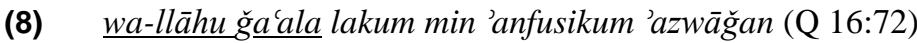

„Gott hat euch aus euch selber Gattinnen gemacht ${ }^{\text {‘22 }}$

16 GILLIOT, Claude, "Narratives". Encyclopedia of the Qur'ān, 3: 521.

17 BoBZIN 2010: 40.

18 PASHOVA 2003: 11-12.

19 HOPPER 1979: 214.

20 BoBZIN 2010: 89.

21 Ebd.: 235

22 Ebd.: 236. 
Die beiden Sätze und die weitere Sätze 16:70, 71,78, 80, 81; 24:45; 37:96 sowie 71:17,19 weisen vier ähnliche Charakteristika auf:

a. Wie bereits erwähnt, stehen die Verben im Perfekt, wobei die Handlung in der Vergangenheit liegt und damit auch abgeschlossen ist.

b. Bis auf zwei Verben (nazzala und faddala in Sure 16:71 und Sure 39:23) haben die Verben die Bedeutung „erschaffen“ bzw. „schöpfen“.

c. Sie stehen nicht im Zusammenhang mit den anderen Versen. In Sure 16 z.B. erscheinen zwei erzählende Abschnitte. In Vers 68 steht „Gott gab der Biene ein“ und in den Versen 74-75 ,stellte er zwei Gleichnisse auf“. Die Wortstellung dieser Verse ist VS, während die Wortstellung in den Versen 65, 70, 71, 72, 78, 80 und $81 \mathrm{SV}$ ist und diese Verse nicht zum Verlauf der erzählenden Abschnitte gehören.

d. Verse, die die Wortstellung SV aufweisen, drücken demgegenüber aus, dass es einzig Gott war, der die Welt erschuf, vgl. die Erklärung bei 'An̄̄s (1966):

wa-qad ğàat hādihī l-'āyātu š-šarīfatu (an-nahl): mufașșilatan li-ni'mi llāhi 'alā nnāsi wa-daf'an li-mā qad yutawahhamu min 'anna llāha šarìkun fìhā 'aw 'anna li-l-

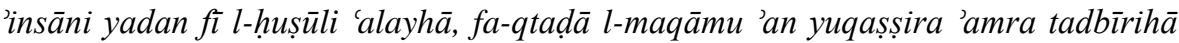

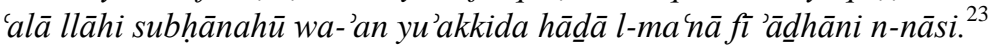

Diese vornehmen Verse erscheinen, um Gottes Gnade den Menschen gegenüber ausführlich darzulegen. Sie weisen zurück, was bereits vermutet wurde, dass Gott in den beschriebenen Handlungen nur ein Teilhaber ist oder dass der Mensch an ihnen teilhat. Die Anfangsstellung des Subjekts „Gott“ beschränkt die Taten auf Gott und möchte dies den Menschen verständlich machen.

Statistisch gesehen (25 VS-Sätze) wird das Subjekt „Gottt“ nachgestellt, wenn das Verb im Perfekt steht und eine abgeschlossene Handlung bezeichnet. In 13 Sätzen steht das Subjekt an der Satzspitze, wobei eine mögliche Erklärung für diese Wortstellung die Betonung ist. Jedoch scheint der Nachdruck dabei nicht die einzige Erklärung zu sein. Die 13 Sätze mit der Wortstellung SV haben parallele Sätze mit den Relativpronomen, z.B.: Sure 2:29 und Sure 14:32:

(9) huwa lladī halaqa lakum mā fì l-'ardi ğamīan (Q 2:29)

„Er ist es, der für euch erschuf, was alles auf der Erde ist“‘24

(10) allāhu llad̄̄ halaqa s-samāwāti wa-l-'arda wa-'anzala mina s-samā̉i māan (Q 14:32)

„Gott ist es, der die Himmel und die Erde schuf und der vom Himmel Wasser herniedersandte ${ }^{\text {c25 }}$

\footnotetext{
23 'ANĪS 1966: 294.

24 BoBZIN 2010: 12.

25 Ebd.: 222.
}

$J A I S \bullet 14$ (2014): 63-86 
Zusätzlich gibt es auch parallele Sätze mit der Wortstellung VS (z.B. Sure 29:44). In diesen Sätzen wird ebenso wie bei den SV-Sätzen zum Ausdruck gebracht, dass Gott der einzige ist, der Himmel und Erde erschuf. Die verschiedenen Satzstrukturen mit ähnlichem Inhalt weisen darauf hin, dass es sich um rhetorische Mittel handelt bzw. dass Wiederholungen von unterschiedlichen Satzbauarten bzw. Wortstellungstypen verhindert werden sollen.

\subsection{Verb im generellen Präsens}

In den folgenden Sätzen findet sich sowohl die Wortstellung VS als auch die Wortstellung SV.

Beispiele:

(11) wa-llāhu yarzuqu man yašāóu bi-ġayri hisābin (Q 2:212)

„Gott versieht mit Gaben, ohne abzurechnen, wen er will“26

(12) yamhaqu llāhu r-ribā wa-yurbī ș-sadaqāti wa-llāhu lā yuhibbu kulla kaffārin 'ațīmin (Q 2:276)

„Gott macht den Zins zunichte, die Almosen vermehrt er. Und Gott liebt keinen Gottlosen, keinen Sünder، 27

(13) $\quad$ wa-llāhu yuhibbu l-muhsiniña $(\mathrm{Q} 3: 134)$

„Gott liebt die, die Gutes tun“28

(14) wa-yatūbu llāhu 'alā man yašăàu wa-llāhu 'alìmun ḥakìmun (Q 9:15)

„Gott wendet sich gnädig zu, wem er will, und Gott ist wissend, weise“‘29

(15) $\quad$ wa-yazìdu llāhu lladīna htadaw hudan (Q19:76)

„Gott bestärkt die, die sich leiten lassen, in der rechten Leitung “630

Reuschel erklärt den Begriff ,generelles Präsens“ folgendermaßen:

Von der Funktion eines nicht-aktuellen bzw. generellen Präs. spricht man dann, wenn durch die entsprechende Verbalform Handlungen bezeichnet werden, die in bezug auf die Zeit unbegrenzt, d.h. an keine Zeitstufe gebunden, aus der Vg (über die Gw) in die Zk laufend, also zeitstufenlos sind. Solche Handlungen können sich auch mit Unterbrechungen ( sich wiederholende, iterative Handlungen) vollziehen. ${ }^{31}$

Die zusammengestellten Verben im generellen Präsens haben zwei Bedeutungen: Entweder behandeln sie die Gottesmacht, oder die Beziehung zwischen Gott und den Gläubigen und den Ungläubigen, bzw. seine Leitung, den Lohn und die Strafe, die sie von Gott bekommen

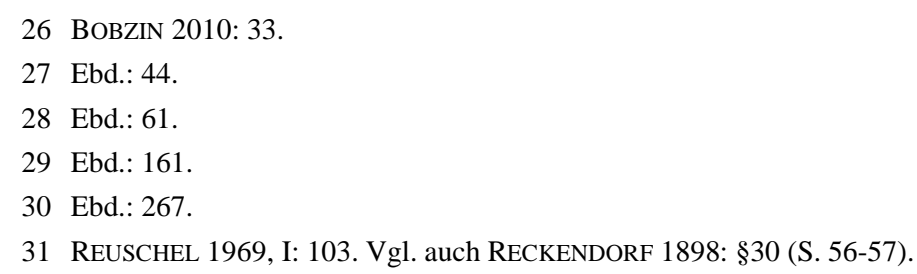


werden. Diese Ideen werden durch unbegrenzte bzw. zeitstufenlose Verbformen ausgedrückt.

Laut Bloch erscheint die Wortstellung SV häufiger als sonst in den Versen der Poesie, die gnomischen Inhalt haben. ${ }^{32}$ Die statistischen Angaben weisen darauf hin, dass im Koran die Wortstellung SV in Sätzen mit dem Subjekt „Gott“ und einem Verb im generellen Präsens häufiger ist. 85 Sätze besitzen die Wortstellung SV im Vergleich zu 35 Sätzen mit der Wortstellung VS. Dazu kommen weitere 34 Sätze mit der Wortstellung VS, deren Verb im Perfekt steht, aber dieselbe Funktion wie das generelle Präsens einnimmt, ${ }^{33}$ z. B.:

(16) wa'ada llāhu l-munāfiqīna wa-l-munāfiqāti wa-l-kuffāra nāra ğahannama hāalidīna fíhā (Q 9:68)

„Den Heuchlern, den Heuchlerinnen und den Ungläubigen - das Feuer der Hölle hat ihnen Gott verheißen, darin sie ewig bleiben“"34

(17) radiya llāhu 'anhum wa-raḍu 'anhu (Q 5:119)

„Gott hat an ihnen Wohlgefallen und sie an ihm“335

Weiter zu beobachten ist die Stellung der Sätze am Ende des Verses oder am Anfang eines neuen Verses. Das Verb und das Subjekt „Gott“ sind nicht die letzten Wörter im Vers. Daher tragen sie keinen Reim, der die Wortstellung beeinflussen könnte. Zusätzlich tragen diese Sätze nichts an neuer Information zu den vorherigen Versen bei.

Gnomisches Präsens ist auch in Adversativsätzen, deren Subjekt „Gott“ ist, zu finden. 10 Sätze besitzen die Wortstellung VS, z.B. Q 2:221.

(18) 'ulāika yad'ūna 'ilā n-nāri wa-llāhu yad'ū 'ilā l-ğannati wa-l-maǵfirati

„Jene laden ein zum Höllenfeuer, doch Gott lädt ein zum Paradiesgarten und zur Vergebung، ${ }^{\text {36 }}$

Adversativsätze gehören zu den Paradebeispielen koranischer Polemik, d.h. die Gegenseitigkeit bzw. der Parallelismus zwischen dem Verhältnis der Ungläubigen zu Gott und dem Verhältnis Gottes zu den Ungläubigen wird zum Ausdruck gebracht. ${ }^{37}$ Fischer erklärt, dass der koordinierte Satz dem Vordersatz nicht immer adäquat, sondern manchmal adversativ ist. Als Beispiel führt er Q 2:232 $2^{38}$ an. ${ }^{39}$ Auch in der modernen arabischen Sprache erscheint die Wortstellung SV in Adversativsätzen. In diesen Fällen sind die Ereignisse nicht chronologisch oder aufeinanderfolgend zu verstehen, sondern simultan. Wenn das Verb in dem Satz eine Aktion, die sich wiederholt, oder eine entstehende

32 BLOCH 1946: 103.

33 REUSCHEL 1969, I: 141, 144.

34 BOBZIN 2010: 167.

35 Ebd.: 108 .

36 Ebd.: 35 .

37 JONES, A., "Polemic". Encyclopaedia of the Qur'ān, 4: 117.

38 wa-llāhu ya lamu wa-'antum lā ta lamūna.

39 FISCHER 1972: §404 (S. 184).

$J A I S \cdot 14$ (2014): 63-86 
Handlung bezeichnet, dann ist die Wortstellung SV üblich. Auch die Wortstellung VS ist zu finden, wenn das Verb im Perfekt steht und damit eine vollständige und nichtkontinuierliche Handlung bezeichnet. ${ }^{40}$

In den folgenden zwei Adversativsätzen ist die Wortstellung VS:

(19) wa-makarū wa-makara llāhu wa-llähu hayru l-mākirīna (Q 3:54)

„Und sie schmiedeten Ränken, und auch Gott schmiedete Ränke“441

(20) wa-yamkurūna wa-yamkuru llāhu wa-llāhu hayru l-mākirīna (Q 8:30)

$\mathrm{Ja}$, sie schmieden ränke und auch Gott schmiedet Ränke “42

In Sure 8:30 ist die VS Wortstellung möglich durch den „Chiasmus“, der besonders in der Poesie eines der beliebtesten Stilmittel ist. ${ }^{43}$ Das Abweichen von der regulären Wortstellung, um einer stilistischen Abwandlung willen, führte zu der Wortstellung VS statt SV, wobei der Vers lauten würde: *wa-yamkurūna wa-llāhu yamkuru wa-llāhu hayru lmākirīna.

Die Wortstellung in Sure 3:54 könnte mit Pashovas Erklärung begündet werden. Das Verb in diesem Vers steht im Perfekt und bezeichnet eine Handlung, die in der Vergangenheit liegt und abgeschlossen ist. In diesem Fall ist die Wortstellung VS üblicher. Mehren führt Sure 3:54 als Beispiel für das ornamentale Mittel der Rede, dass Zeugma heißt. Er erklärt diesen Begriff folgendermaßen: , المُششاكلة, das Zeugma, besteht darin, dass man einen Begriff durch einen ihm nicht zukommenden Ausdruck bezeichnet, weil er mit diesem in Verbindung kommt, z.B. Sure 3:47, wo der auf Gott an und für sich nicht anwendbare Begriff des Ränkeschmiedens wegen der Verbindung mit dem Vorhergehenden 

Unter dieser Kategorie also erscheinen Sätze, die sich zwar syntaktisch und inhaltlich unterscheiden und dennoch die o.g. Gemeinsamkeiten tragen. Das lässt darauf schließen, dass alle Verse dieselbe Funktion bzw. Intention haben und zwar Gottes Allmacht und seine Huld auszudrücken. Diese These lässt sich an zwei Beobachtungen festmachen:

1. Parallele Sätze: Zwischen den Versen sind ähnliche Sätze zu finden, aber mit unterschiedlicher Wortstellung z.B.:

(21) yahdī llāhu li-nūrihī man yašāàu (Q 24:35)

„Gott leitet, wen er will, zu seinem Licht ${ }^{\star 45}$

(22) wa-llāhu yahdī man yašăảu iilā șirāṭin mustaqīmin (Q 2:213)

„Gott leitet, wen er will, auf einen geraden Weg ${ }^{\text {‘46 }}$

\footnotetext{
40 PASHOVA 2003: 16.

41 BobZIN 2010: 53.

42 Ebd.: 153.

43 BLOCH 1946: 37.

44 MEHREN 1853: 103.

45 BobZIN 2010: 308.

46 BoBZIN 2010: 33.
} 
(23) yurīdu llāhu li-yubayyina lakum wa-yahdiyakum sunana llad̄ina min qablikum wayatūba 'alaykum (Q 4:26)

„Gott will für euch Klarheit schaffen und euch nach dem Brauche derer, die vor euch lebten, leiten und sich euch zuwenden“"47

(24) wa-llāhu yurīdu 'an yatūba 'alaykum (Q 4:27)

„Gott will sich euch zuwenden“48

(25) yurīdu llāhu 'an yuhaffifa 'ankum (Q 4:28) ${ }^{49}$

„Gott will es euch leicht machen“50

In der Einleitung zu diesem Aufsatz wurde erwähnt, dass Suleiman die Wortstellung SV auf den gewünschten Effekt der Betonung zurückführt. Mit Suleimans Deutung lassen sich zwar alle Sätze in diesem Kapitel, die die Wortstellung SV besitzen, erklären - durch die Voranstellung des Subjekts „Gott“ wird betont, dass nur Gott die erwähnte Handlung in jedem Vers durchführen kann - aber wird diese Idee nicht auch durch Sätze mit der Wortstellung VS, wie z.B. Q 24:35, ausgedrückt? Diese Frage wäre zu bejahen.

Auch angesichts der folgenden Sätze stellt sich die Frage, ob hier tatsächlich ein Unterschied bzw. eine andere Intention vorliegt:

(26) wa-llāhu yuhibbu l-muhsinīna (Q 3:134)

„Gott liebt die, die Gutes tun“651

(27) ¿inna llāha yuhibbu l-muhsinīna (Q 5:13)

„Siehe, Gott liebt diejenigen, die Gutes tun“652

(28) wa-llāhu yarzuqu man yašā'u bi-ġayri hisābin (Q 2:212)

„Gott versieht mit gaben, ohne abzurechnen, wen er will“53

(29) 'inna llāha yarzuqu man yašāu bi-gayri hisābin (Q 3:37)

„Siehe, Gott versieht mit gaben, wen er will, ohne abzurechnen“654

Diese vier Beispiele unterliegen zwar zwei verschiedenen Satzbaumustern, nämlich einerseits SV-Sätzen und andererseits Sätzen, die durch die Betonungspartikel 'inna eingeleitet werden. Aber sie sind inhaltlich identisch, wobei die unterschiedliche Satzstruktur als Stilmittel zur Variation des Ausdrucks verstanden werden kann.

47 Ebd.: 73.

48 Ebd.: 74.

49 Hinter der Wortstellung VS-SV-VS in Sure 4:26-28 könnte die Intention stehen, die Wiederholung derselben syntaktischen Struktur zu vermeiden.

50 BoBZIN 2010: 74.

51 Ebd.: 60.

52 Ebd.: 95.

53 Ebd.: 33.

54 Ebd.: 51.

$J A I S \bullet 14$ (2014): 63-86 
2. Klauseln: Die zweite Beobachtung bezieht sich auf ein Beispiel verschiedener Satzstrukturen, welche die gleiche Funktion bzw. eine Idee ausdrücken - die Klauseln. Bell beschreibt die unterschiedlichen Strukturen und Stilmittel im Koran. Eine dieser Strukturen nennt sich 'Rhyme-Phrase' oder 'Reimsätze'. Aussagen, die Gott betreffen bzw. beschreiben, erscheinen oft am Ende des Verses, besonders in den langen Suren. Falls der Vers kurz ist, bildet das Wort oder der Satz, die den Reim tragen, den Integralteil der grammatikalischen Struktur. Sie sind nötig für die Bedeutung des Verses. In anderen Fällen stehen diese Sätze nicht in Zusammenhang mit den vorherigen Versen. Dennoch stören sie nicht die Bedeutung des vorherigen Satzes. Diese Reimsätze tragen unterschiedliche Endreime wie $\bar{i} m$, z.B.:

(30) wa-llāhu 'alīmun hakìmun (Q 8:71)

„Gott ist wissend, weise“

Die Klauseln werden benutzt, um eine Erlösungsbotschaft abzuschließen. Sie sind notwendig, um die Wahrheit zu verbreiten und funktionieren als Kehrreim. ${ }^{56}$

Ebenso wie die Reimsätze verschiedene Strukturen haben, aber eigene Funktion besitzen, weisen die beschriebenen Strukturen in dieser Kategorie verschiedene Arten des Aufbaus und unterschiedliche Wortstellungen auf.

\subsection{Verben im Imperfekt}

In den folgenden Sätzen bezeichnet das Verb eine zukünftige Handlung, die am jüngsten Tage geschehen wird. Die Wortstellung in diesen Sätzen ist VS:

(31) wa-yas'alūnaka 'ani l-ğibāli fa-qul yansifuhā rabbī nasfan (Q 20:105)

„Sie fragen dich nach den Bergen. Sprich: Mein Herr wird sie zu Staub zerstäuben ${ }^{457}$

In einem Satz ist die Wortstellung SV:

(32) quli llāhu yuhȳ̄kum tumma yumītukum țumma yağma'ukum ilā yawmi l-qiyāmati lā rayba fihi (Q 45:26)

„Sprich: Gott gibt euch das Leben, dann lässt er euch sterben, dann versammelt er euch zum Tag der Auferstehung, über den kein Zweifel herrscht ${ }^{\star ‘ 58}$

Durch die Wortstellung SV in Sure 45:26 wird betont, dass nur Gott wiederbelebt, sterben lässt und die Menschen am jüngsten Tage versammelt. Ebenso wie bei den SV-Sätzen in Abschnitt 1.2 gibt es auch einen parallelen Satz mit dem Relativpronomen llad $\underline{\imath}$ :

(33) huwa lladī yuhȳ̄ wa-yumìtu (Q 40:68)

„Er ist es, der lebendig macht und sterben lässt ${ }^{\star 59}$

55 BoBZIN 2010: 157.

56 BELL 1953: 70.

57 BobZIN 2010: 275.

58 Ebd.: 443 
Yehudit Dror

\section{Satzart}

\subsection{Negative Sätze}

Es finden sich 13 Negativsätze mit der Wortstellung VS:

(34) wa-lā yukallimuhumu llāhu yawma l-qiyāmati wa-lā yuzakkīhim (Q 2:174)

„Gott wird am Tag der Auferstehung nicht zu ihnen sprechen und sie auch nicht läutern“"60

Es gibt weitere negative Sätze, deren Wortstellung SV ist, und deren Verb im generellen Präsens steht ${ }^{61}$ :

(35) wa-llāhu lā yahdī l-qawma z-zālimīna (Q 2:258)

„Gott leitet kein frevlerisches Volk“662

Zwischen den zwei Satztypen $(l \bar{a}+\mathrm{SV}$ und $l \bar{a}+\mathrm{VS})$ bestehen die folgenden Unterschiede:

Erstens erscheinen die SV-Sätze am Ende des Verses, wobei das letzte Wort den Reim trägt. VS-Sätze erscheinen am Anfang, in der Mitte und am Ende des Satzes. In Sure 18:49 und Sure 20:52 steht der Satz am Ende des Verses und das letzte Wort reimt sich.

Zweitens sind die SV-Sätze syntaktisch und gedanklich ähnlich, denn die Verben und die Objekte wiederholen sich. In diesen Versen geht es um das Verhältnis Gottes zu den Ungläubigen. Die Verben der VS-Sätze sind gedanklich verschieden. Es geht um Gottes Macht, sein Verhältnis zu den Ungläubigen und die Führung Gottes.

Drittens tragen die SV-Sätze keine neue Information zu den vorherigen Sätzen bei, die syntaktisch und inhaltlich vollständig sind. Ganz im Gegensatz dazu enthalten die VSSätze die Hauptinformation des Verses.

Die Wortstellung SV erscheint in zwei weiteren Sätzen:

(36) wa-mā llāhu yurīdu zulman li-l-'álamīna (Q 3:108)

„Gott will nicht, dass den Weltbewohnern Unrecht geschieht"“63

(37) wa-mā llāhu yurīdu zulman li-l-ibādi (Q 40:31)

„Gott will nicht, dass seinen Knechten Unrecht geschieht“664

Eine Erklärung für die Wortstellung SV in Negativsätzen gibt Ğurğān̄i:

'idā qulta mā fa'altu kunta nafayta 'anka fi lan lam yațbut 'annahū maf'ūlan wa-id qulta mā 'anā fa'altu kunta nafayta 'anka filan tabata 'annahu maf'ūlun. tafsīr d̄ālika 'annaka 'id̄ā qulta: mā qultu hādihī kunta nafayta 'an takūna qad qulta d̄āka wa-kunta nūzirta fì šay'in lam yațbut 'annahū maqūlun. wa-'idā qulta: mā 'anā qultu

59 BobZIN 2010: 418.

60 Ebd.: 28.

61 Diese Sätze wurden in Kategorie 2, Verben im generellen Präsens klassifiziert.

62 BobZIN 2010: 42.

63 Ebd.: 58.

64 Ebd.: 415.

$J A I S \cdot 14$ (2014): 63-86 


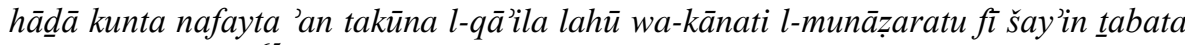
'annahū maqūlun. ${ }^{65}$

Im Satz $m \bar{a}$ fa'altu wird eine Handlung verneint, wobei nicht sicher ist, ob die Tätigkeit tatsächlich stattfand. Im Gegensatz dazu ist sie im Satz $m \bar{a}$ 'ana fáaltu sicher. Ein Beispiel dafür ist: wenn jemand den Satz mā qultu hād $\bar{a}$ sagt, so wird die Handlung bzw. das Gesagte verneint. Im Satz $m \bar{a}$ 'anā qultu verneint jemand, dass er der Täter ist, wobei das Gesagte stattfand.

In den beiden Sätzen in Sure 3:108 und Sure 40:31 wird also verneint, dass Gott der Täter ist, d.h. nicht er will, dass irgend jemandem in der Welt Unrecht geschieht.

\subsection{Fragesätze}

In Fragesätzen ist das Subjekt „Gott“ nachgestellt:

(38) Kayfa yahdī llāhu qawman kafarū ba'da ìmānihim (Q 3:86)

„Wie soll Gott Menschen führen, die ungläubig wurden, nachdem sie gläubig waren ${ }^{\prime 666}$

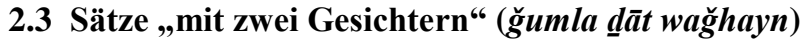

Ein Satz „mit zwei Gesichtern“ wird erklärt folgendermaßen: „Das natürl. Subj. des Satzes an die Spitze gestellt, die Konstruktion hierauf abgebrochen, und in dem erneut begonnenen Satze mittels eines rückweisenden Pronomens die Beziehung zu dem natürl. Subj. hergestellt. Das isolierte Subj. ist ein مبتدأ der Rest des Satzes dessen خبطر Räckweisepron. heißt رابط ذات جملة كبرى هor der hinter den isolierten Satzteilen stehende Satz «ein kleiner Satz» رغرى (197)

Reckendorf (1898) und Bloch (1946) sprechen von einer sog. Isolierung des natürlichen Subjekts. Das erste Wort oder die erste Information des Satzes ist das, was für den Sprecher und für die anderen bekannt ist, und wird als das natürliche Subjekt bezeichnet. Daher ist der Hörer anschließend für die neue Information offen. Dem natürlichen Subjekt folgt das natürliche Prädikat. Das Fortschreiten im Satz von natürlichem Subjekt zu natürlichem Prädikat vollzieht sich normalerweise fließend, aber manchmal gibt es eine Unterbrechung zwischen den beiden Teilen, indem das natürliche Subjekt isoliert ist. ${ }^{68}$

Im folgenden Beispiel liegt die Aufmerksamkeit bzw. der Nachdruck auf dem isolierten Subjekt bzw. auf dem Demonstrativpronomen. Es bezieht sich auf diejenigen, die glauben,

65 ĞURĞĀNī (o.J.): 96.

66 BOBZIN 2010: 56

67 RECKENDORF 1921: §182 (S. 366).

68 RECKENDORF 1898: §257, S. 782-783. Reckendorf führt verschiedene Beispiele der Isolierung an, wie: lākini r-rāsihūna fì l-ilmi minhum wa-l-mu'minūna yu'minūna bi-mā 'unzila 'ilayka wa-mā 'unzila min qablika wa-l-muqūmīna ș-șalāta wa-l-mu tūna z-zakāta wa-l-mu'minūna bi-llāhi wa-l-yawmi l-āhhiri ủāōika sa-nu'tīhim 'ağran 'azīman (Q 4:162); wa-l-qamara qaddarnāhu manāzila hattā 'āda ka-lurğūni l-qadìmi (Q 36:39); fa-'ammā l-yatìma fa-lā taqhar (Q 93:9) 
rechtgeleitet sind, Gott und seinem Gesandten gehorchen, umkehren und gute Werke verrichten. Der „kleine Satz“ beschreibt ihren Lohn: Gott wird sich ihrer erbarmen, er verzeiht ihnen und tauscht ihre schlechten Taten gegen gute ein:

(39) wa-l-mu'minūna wa-l-mu'minātu ba'duhum 'awliyā'u ba'ḍin ya'murūna bi-l-ma'rüfi wa-yanhawna 'ani l-munkari wa-yuqūmūna ș-șalāta wa-yu'tūna zakāta wa-yuțīūna llāha wa-rasūlahù ủūàika sa-yarhamuhumu llāhu inna llāha 'azīzun hakīmun (Q 9:71)

„Die Gläubigen, die Männer wie die Frauen, die stehen einander bei. Sie gebieten das Rechte und verbieten das Schlechte, sie verrichten das Gebet und entrichten die Armensteuer, und sie gehorchen Gott und seinem Gesandten. Sie sind es, derer sich Gott erbarmen wird. Siehe, Gott ist mächtig, weise.“699

\section{Einleitungspartikel und Pronomen}

\subsection{Die Partikel sa, sawfa, qad, la-qad}

Die Wortstellung nach diesen Partikeln ist VS:

(40) sa-yudhiluhumu llāhu fì rahmatihī (Q 9:99)

„Gott wird sie in sein Erbarmen aufnehmen“،70

(41) wa-sawfa yunabbi'uhumu llāhu bi-mā kānū yașna üna (Q 5:14)

„Gott wird ihnen dann kundtun, was sie je angerichtet haben“"71

(42) qad ya lamu llāhu l-mu'awwiqina minkum (Q 33:18)

„Gott kennt die Verhinderer unter euch“"72

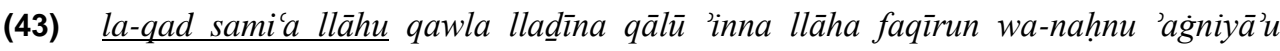
(Q 3:181)

„Gott hat gehört die Rede, derer, welche sprachen: Siehe, Gott ist arm, und wir sind reich" ${ }^{\text {c73 }}$

\subsection{Die Partikel tumma}

Nach dieser Partikel ist die Wortstellung VS und die Wortstellung SV zu finden:

(44) tumma 'anzala llāhu sakīnatah̄u 'alā rasūlihī wa-'alà l-mu'minīna wa-'anzala

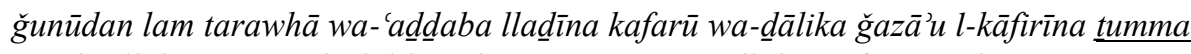

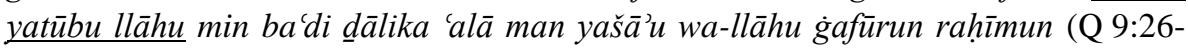
27)

69 BobZin 2010: 168.

70 Ebd.: 171.

71 Ebd.: 95.

72 Ebd.: 367.

73 Ebd.: 66.

JAIS • 14 (2014): 63-86 
„Da sandte Gott hernieder seine < Ruhe>, auf seinen Gesandten und die Gläubigen, und schickte Heerscharen herab, die ihr nicht saht, und bestrafte die Ungläubigen. Das ist der Lohn derer, die nicht glauben. Danach aber wendet sich Gott gnädig zu, wem er will. Gott ist bereit zu vergeben, barmherzig. “"74

(45) qul sìrū fì l-’arḍi fa-nzurū kayfa bada'a l-halqa tumma llāhu yunšìu n-našsata l'āhirata 'inna llāha 'alā kulli šay'in qadīrun (Q 29:20)

„Sprich: Zieht im Land umher, und schaut, wie er Schöpfung zum ersten Mal vollbracht hat! Darauf bringt Gott hervor die letzte Schöpfung. Siehe, Gott ist aller Dinge mächtig، ${ }^{75}$

Die Partikel tumma ist eine Verknüpfungspartikel, deren Funktion ist, die Handlungen zu ordnen:

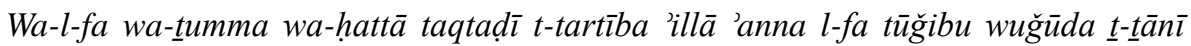
ba'da l-'awwali bi-gayri muhlatin wa-tumma tūğibuhu bi-muhlatin wa-li-dālika qāla Sībawayhi marartu bi-rağulin țumma mra'atin fa-l-murūru hunā murūrāni. ${ }^{76}$

$\underline{t} u m m a, f a$ - und ḩattā sind Anordungspartikel. Nach der Partikel fa- kommen die Handlungen gleich nach einander ohne Pause. Nach tumma ist eine Pause zu denken. Darum sagte Sībawayhi: In dem Satz „Ich bin an einem Mann vorbeigegangen und dann an einer Frau" hat ein Vorübergehen zweimal stattgefunden.

In VS-Sätzen nach der Partikel tumma folgen zwar die beschriebenen Handlungen zeitlich aufeinander; aber es kann auch der Fall sein, dass es eine kleinere oder größere Zeitspanne zwischen den Ereignissen liegt, wie man in Sure 9:25-27 sieht:

Gott hat euch (doch) an vielen Orten zum Sieg verholfen [...] Dann sandte Gott seine sakīna auf seinen Gesandten und auf seine Gläubigen herab [...] Hierauf, nachdem dies (alles) geschehen ist, wendet sich Gott (gnädig) wieder zu, wem er will. ${ }^{77}$

Der Zusammenhang zwischen Wortstellung und zeitlicher Aufeinanderfolge im Koran ist mit der entsprechenden Situation im Alten Testament vergleichbar. Dieser Aspekt des Alten Testaments wurde von John Myhill untersucht. In der Einleitung zu seinem Artikel schreibt er:

VS word order is statistically correlated with temporally sequenced clauses, while $\mathrm{SV}$ word order is associated with unsequences clauses [...] A temporally sequenced ${ }^{78}$ clause is one which advances the time reference of a narrative (for example: Genesis $\left.1: 3-5^{79}\right) .^{80}$

\footnotetext{
74 BoBZin 2010: 162.

75 Ebd.: 346.

76 ZAMAHEŠARĪ 1879: 141

77 PARET 1966: 153.

78 MYHILL erwähnt, dass der Begriff "temporally sequenced clause" parallel zu den Begriffen "narrative clause" oder "forgrounded clause" verwendet wird.

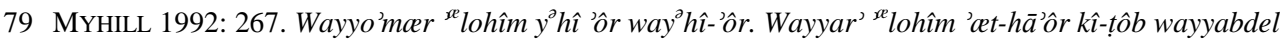


Wie bereits erwähnt, ist die Wortstellung SV ebenso nach der Partikel tumma zu finden. Sie verbindet zwei Sätze, die in konzessivem Vehältnis zueinander stehen:

(46) [...] lā tasfikūna dimāaakum wa-lā tuhriğūna 'anfusakum min diyārikum tumma 'aqrartum wa-'antum tašhadūna tumma 'antum häulà'i taqtulüna 'anfusakum watuhriǧūna farīqan minkum min diyārihim tazāharūna 'alayhim bi-l-ìtmi wa-l'udwāni (Q 2:84-85)

„[...] Ihr sollt nicht gegenseitig euer Blut vergießen und nicht einander aus euren Wohnstätten vertreiben [...] Dennoch tötet gerade ihr einander und vertreibt einen Teil von euch aus ihren Wohnstätten." 81

Doch in Sure 29:20 (s.o.), wo das Subjekt „Gott“ ist, besteht kein konzessives Verhältnis, sondern tumma verbindet zwei Handlungen, den Anfang der Schöpfung und das Schaffen einer letzten Existenz, die einander folgen. Aus diesem Grund sollte die Wortstellung VS sein und nicht SV. Dabei wird aber betont, dass Gott derjenige ist, der die letzte Existenz schafft, daher wird das Subjekt hervorgehoben.

\subsection{Die Partikel bal}

Ebenso wie bei der Partikel tumma hat die Partikel bal mehrere Funktion. Wenn bal als Adversativpartikel dient, ist die Wortstellung VS:

(47) 'a-fa-kullamā ğ 'àakum rasūlun bi-mā lā tahwā 'anfusukumu stakbartum fa-farīqan

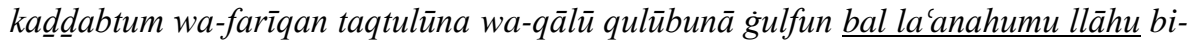
kufrihim fa-qalīlan mā yu'minūna (Q 2:87-88)

„Aber waret ihr (Juden) denn nicht jedesmal, wenn ein Gesandter euch etwas überbrachte, was nicht nach eurem Sinn war, hochmütig und erklärtet ihn für lügnerisch oder brachtet ihn um? Und sie sagen: Unser Herz ist (eben) unbeschnitten. Aber nein! [bal ,und“, d.h. dennoch - Y. Dror] Gott hat sie (zur Strafe) für ihren Unglauben verflucht. Darum sind sie so wenig gläubig. “82

Die Funktion der Partikel bal als Adversativpartikel wurde der hebraistischen Forschung entnommen. Eitan (1929) vergleicht zwischen אבל uبَ im Rahmen seines Artikels "Hebrew and Semitic Particles: Comparative Studies in Semitic Philology". Er führt aus, dass im späteren und heutigen Hebräisch diese Partikel nur als Adversativpartikel dient, während sie im alten oder biblischen Hebräisch verwendet wurde, um eine Idee zu bestätigen oder um etwas zu erklären:

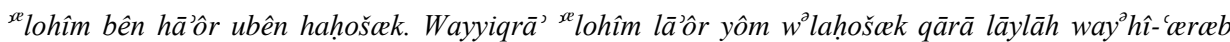
way hî-boqar yôm 'āhād. „Da sprach Gott: Es werde hell, und es wurde hell. Und Gott sah die Helle, dass sie gut war. Da schied Gott zwischen der Helle und dem Dunkel. Und Gott nannte die Helle Tag, das Dunkel aber nannte er Nacht. Da ward aus Abend und Morgen der erste Tag [LUTHER]“ (Gen 1: 3-5).

80 MYHILL 1992: 265.

81 KHOURY 2004, II: 36. Vgl. BobZIN 2010: 18.

82 PARET 1966: 15.

$J A I S \cdot 14$ (2014): 63-86 
The current comparison of the latter (אבל) with the Arabic conj. بَّ is certainly correct when applied to the adversative אבל 'but', 'howbeit', as it appears in the later books of the Bible (Ezra, Daniel, Chronicles) and in the general use of אבל [... = Arab. أَفَكاكَ, However, the foregoing identification with Arab bal appears artificial when referring to the asseverative אבל , 'verily', as is used in older Hebrew. Compare Gen. 17:19 ${ }^{83}$, 1Kings 1:43. ${ }^{84}$

This אבל 'verily', employed to introduce independent sentences with asseverative force, seems to differ both from the absolute Mish. אבל = Arab. بَلَى and from the adversative אבל 'but', 'except that', 'howbeit'.

Bal als Betonungspartikel ist in Sätzen mit der Wortstellung SV zu beobachten, wobei diese Sätze mit den Versen aus dem Alten Testament (s.o.) vergleichbar sind:

(48) 'a-lam tara 'ilā llad̄ina yuzakkūna 'anfusahum bali llāhu yuzakkī man yašă'u wa-lā yuẓlamūna fatīlan (Q 4:49) ${ }^{86}$

„Sahst du denn die nicht, die sich selber für rein erklären? Nein, Gott erklärt für rein, wen er will, und ihnen wird kein Quentchen Unrecht getan. “67

\subsection{Die Partikel $f a$ -}

Die Sätze, deren Subjekt „Gott“ ist und die durch die Partikel $f a$ - eingeleitet werden, lassen sich in vier Gruppen einteilen:

1. VS-Sätze, die in erzählenden Abschnitten erscheinen, die eine Abfolge von Geschehnissen schildern:

(49) fa-stağāba lahū rabbuhū fa-șarafa 'anhu kaydahunna 'innahū huwa s-samīu l'alīmu (Q 12:34)

„Da erhörte ihn sein Herr und wendete ihre Ränkespiele von ihm ab. Siehe, er ist der Hörende, der Wissende ${ }^{\text {88 }}$

2. VS-Sätze, wo ein Konsekutivverhältnis besteht. In diesen Sätzen geht es um die Reaktion Gottes bzw. seine Vergeltung der Handlungen der Ungläubigen. Die Sätze nach fa drücken die Folge ihers Fehlverhaltens aus, nämlich die Strafe Gottes:

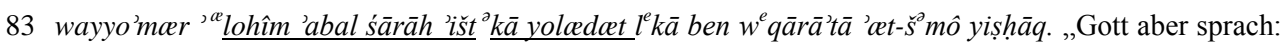
Nicht doch; Sara, dein Weib, gebärt dir einen Sohn, den sollst du Jizhak nennen.“

84 wayya'an yônātān wayyo'mar la ${ }^{x}$ doniyyāhû 'abal ${ }^{a}$ doniyyāhû hammalak-dāwid himlîk 'at-šs lomoh. „Da antwortete Jonathan und sprach zu Adonijahu: Nicht doch! Unser Herr, der König David, hat Salomo zum König erklärt.“"

85 EITAN 1929: 206. Vgl. auch MURAOKA 1985: 129.

86 PARET 1966: 71 übersetzt die Partikel bal mit „Vielmehr“: „Hast du nicht jene gesehen, die sich selber für rein erklären? Vielmehr erklärt Gott für rein, wen er will. Und ihnen wird nicht ein Fädchen unrecht getan.

87 BobZIN 2010: 76.

88 Ebd.: 204. 
(50) Ka-da'bi 'āli fir'awna wa-llad̄iña min qablihim kaḍdabū bi-'āyātinā fa-'ahadahumu

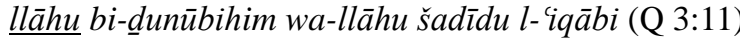

„Wie den Leuten Pharaos und denen, die vor ihnen lebten: Sie erklären unsere Zeichen für Lüge, und da [daher] kam Gott (mit einem Strafgericht) über sie (zur Vergeltung) für ihre Schuld. “' ${ }^{\text {(89 }}$

3. VS-Sätze, deren Verb im generellen Präsens steht:

(51) fa-yudillu llāhu man yaš̄̄̄u wa-yahdī man yašā'u (Q 14:4)

„So führt Gott in die Irre, wen er will und führt auf dem rechten Wege, wen er will"“90

4. SV-Sätze:

(52) fa-llāhu yaḥkumu baynahum yawma l-qiyāmati fi-mā kānū fìhi yah̆talifūna (Q 2:113, $4: 141)$

„Doch Gott wird zwischen ihnen richten am Tag der Auferstehung in dem, worin sie uneins waren. “91

Beide Satztypen finden sich in Sure 22: 69:

(53) allāhu yahkumu baynakum yawma l-qiyāmati fì-mā kuntum fìhi tahtalifüna „Gott richtet zwischen euch am Tag der Auferstehung in dem, worin ihr uneins wart. “92

Der einzige Unterschied zwischen Q 22:69, 2:113 und 4:141 ist, dass der Satz llāhu yahkumu in Sure 22:69 am Anfang des Verses steht, während der Satz in Sure 2:113 und Sure 4:141 in der Mitte des Satzes steht, wo eine Pause beim Lesen angezeigt ist. In den drei Sätzen liegt der Nachdruck auf dem Subjekt allāh.

\subsection{Das Bestimmungsadverb ka- $\underline{d} a \overline{l i k a}$}

In 39 Sätzen herrscht die Wortstellung VS vor. Unter diesen Sätzen befinden sich 18 Sätze, deren Subjekt „Gott“ ist:

(54) ka-dālika yadribu llāhu l-ḥaqqa wa-l-bātila fa-'ammā z-zabadu fa-yad̆habu ğufā'an wa-'ammā mā yanfa'u n-nāsa fa-yamkutu fì l-'arḍi ka-d̄alika yadribu llāhu l-'amtāala (Q 13:17)

„So verdeutlicht Gott das Wahre und das Nichtige. Was nun den Schaum betrifft, geht er dahin - nutzloses Treibgut! Doch was den Menschen nützt, bleibt auf der Erde. So prägt Gott die Gleichnisse.“، $₫ 33$

Nur zwei Sätze besitzen die Wortstellung SV:

89 PARET 1966: 44 (das Wort „Daher“ erscheint nicht in Parets Übersetzung). Vgl. BoBZIN 2010: 48.

90 BoBZIN 2010: 219.

91 Ebd.: 21.

92 Ebd.: 294.

93 BoBZIN 2010: 215.

JAIS • 14 (2014): 63-86 
(55) qāla rabbi 'annā yakūnu lì ġulāmun wa-qad balaġan̄̄ l-kibaru wa-mra'atī 'āqirun qāla ka-d̄alika llāhu yaf́alu mā yašă'u (Q 3:40)

„Er sprach: Mein Herr, wie soll mir denn ein Knabe werden, wo mich das Alter schon erreichte und meine Frau unfruchtbar ist? Er sprach: So ist Gott. Er tut was er will." "94

(56) qālat rabbi 'annā yakūnu lī waladun wa-lam yamsasnī bašarun qāla ka-dālika llāhu yahluqu mā yašă u (Q 3:47)

„Sie sprach: Mein Herr, wie soll ich denn ein Kind bekommen, wo mich kein menschlich Wesen je berührte? Er sprach: So ist Gott. Er erschafft, was er will“95

Der Unterschied zwischen den Satztypen VS und SV liegt in der Funktion des Bestimmungsadverbs ka-da ālika. In den Sätzen mit der Wortstellung VS ist ka-dāalika eine Bestimmungspartikel. Welche Funktion dieses Pronomens in den SV-Sätzen hat, ist aus Zamahšarīs Exegese ersichtlich:

(ka-dālika) 'ay yaf'alu llāhu mā yašāà min-a l-'af'āli l-'ağībati mițla dālika l-fi lu wa-huwa halqu l-waladi bayna š-šayhi l-fānì wa-l-'ağūzi l-'āqiri 'aw ka-d̂ālika llāhu mubtada' wa-habar 'ay 'alā naḥwi hādihi ș-șifati wa-yaf'alu mā yašā'u bayānun lahu

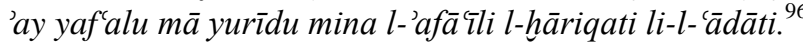

$k a-\underline{d} a \bar{l} l i k a$ heißt: Gott bewirkt die wunderbaren Taten, die er will, wie diese Tat der Erschaffung eines Kindes zwischen einem alten (entkräfteten) Mann und einer un-

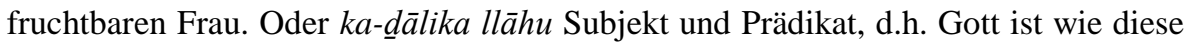
Eigenschaft, wobei der Satz yaf'alu mā yašă $u$ (er tut, was er will) eine Erklärung (bayān) für den vorherigen Satz wäre, d.h. Gott tut die ungewöhnlichen Taten, die er will.

Nach dieser Erklärung gibt es zwei Möglichkeiten, Vers 40 und 43 zu lesen.

1. Die erste Möglichkeit ist:
ka- $\underline{\text { dàlika }}$
llāhu ya'malu mā yašă'u
'ism 'išāra
ğumla 'ismiyya

Hier würde die Übersetzung lauten: „So ist es. Gott tut, was er will.“

2. Die zweite Möglichkeit ist, den letzten Teil des Verses in zwei Sätze einzuteilen:

\begin{tabular}{|c|c|c|}
\hline $\begin{array}{l}\text { ka-d्dālika } \\
\text { mubtada' }\end{array}$ & $\begin{array}{l}\text { llāhu } \\
\text { habar }\end{array}$ & $\begin{array}{l}\text { ya'malu } \\
\text { fi'l } 1+\text { fấcil }^{2}\end{array}$ \\
\hline
\end{tabular}

Die Übersetzung wäre: „So ist Gott. Er tut, was er will.“

Die Möglichkeit, ka-dâlika als Bestimmungsadverb und als Subjekt zu analysieren, besteht nicht in den VS-Sätzen, z.B:

94 Ebd.: 51.

95 Ebd.: 52.

96 ZAMAHֵŠARĨ 1948, I: 322. 
ka-d̄ālika yağ́alu llāhu r-riǧsa 'alā llad̄ina lā yu’minūna (Q 6:125)

„So macht Gott die, die nicht glauben, unrein." ${ }^{\prime 97}$

Die syntaktische Analyse dieses Satzes ist:

$\begin{array}{lll}k a-\underline{d} \bar{a} l i k a & \text { yağ́ălu llāhu } & \text { r-riğsa } \\ \text { 'ism 'išāra } & \text { fi'l+fā'il } & \text { maf'ūl }\end{array}$

Die folgende Analyse, in der das Demonstrativpronomen als Subjekt analysiert wird, ist syntaktisch nicht korrekt:
ka-d̄ālika yağ́alu
llāhu r-riğsa
mubtada'+ habar
mubtada'+ maf'ūl bihi (Das Prädikat fehlt)

\subsection{Die Verwendung des Subjekts „Gott“ statt eines Personalpronomens}

Zuletzt ist Sure 5:67 zu erwähnen, wo das Subjekt „Gott“ statt eines Personalpronomens benutzt wird: ${ }^{98}$

(57) $y \bar{a}$-'ayyuhā r-rasūlu ballig mā 'unzila 'ilayka min rabbika wa-'in lam taf'al fa-māa ballag̀ta risālatahū wa-llāhu ya simuka mina n-nāsi inna llāha lā yahdī l-qawma lkāfirīna

„Gesandter! Übermittle du, was zu dir herabgesandt wurde von deinem Herrn! Wenn du es nicht tust, dann hast du deine Botschaft nicht erfüllt. Und Gott wird dich vor den Menschen beschützen. Siehe, Gott leitet die ungläubigen Menschen nicht recht. “99

Dieser Satz ist nicht anders zu beurteilen als Sätze, in denen sich die Personalpronomina auf Gott beziehen und auf ihnen der Nachdruck liegt, z.B.:

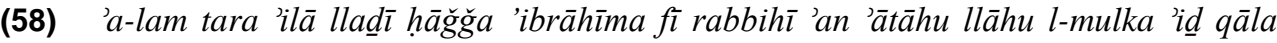
'ibrāhīmu rabbiya llad̄̄ yuhȳ̄ wa-yumītu qāla 'anā 'uhȳ̄ wa-'umītu (Q 2:258)

„Sahst du denn den nicht, der sich mit Abraham über seinen Herrn stritt - dass Gott die Herrschaft ihm gegeben habe? Damals, als Abraham sprach: Mein Herr ist's, der lebendig macht und tötet, sprach er: Ich bin es, der lebendig macht und tötet!‘ ${ }^{100}$

(59) huwa yuhȳ̄ wa-yumītu wa-ilayhi turğa ūna (Q 10:56)

„Er macht lebendig und lässt sterben, und zu ihm werdet ihr zurückgebracht.“" ${ }^{101}$

\footnotetext{
97 BOBZIN 2010: 123.

98 HALEEM 2004: 185. Der Satz sollte lauten: yā-'ayyuhā r-rasūlu ballig mā 'unzila 'ilayka min rabbika wa-'in lam taf'al fa-mā ballagta risālatahū wa-huwa ya șimuka mina n-nāsi

99 BOBZIN 2010: 102.

100 Ebd.: 41.

101 BoBZIN 2010: 182
}

$J A I S \bullet 14$ (2014): 63-86 


\section{Zusammenfassung}

Die folgende Tabelle gibt eine statistische Übersicht über allen untersuchten Verbalsätze, deren Subjekt „Gott“ ist:

\begin{tabular}{lcc}
\hline Untersuchte Struktur & \multicolumn{2}{c}{ Wortstellung } \\
& $S V$ & $V S$ \\
\hline 1. Subjekt + Verb im Perfekt & 13 & 25 \\
2. Subjekt + Verb im Imperfekt & 1 & 3 \\
3. Subjekt + Verb im generellen Präsens & 85 & 35 \\
4. Subjekt + Verb im Perfekt, das dieselbe Funktion des & & 34 \\
generellen Präsens hat & & \\
5. Negativsätze & 2 & 13 \\
6. Fragesätze & - & 7 \\
7. Satz „mit zwei Gesichtern“ (betrifft den „kleinen Satz“) & & 2 \\
8. Sätze nach der Partikel sa- & - & 3 \\
9. Sätze nach der Partikel sawfa & - & 4 \\
10. Sätze nach der Partikel qad & - & 10 \\
11. Sätze nach der Partikel la-qad & - & 7 \\
12. Sätze nach der Partikel tumma & 1 & 5 \\
13. Sätze nach der Partikel bal & 1 & 1 \\
14. Sätze nach der Partikel fa- & 2 & 11 \\
15. Sätze nach dem Bestimmungsadverb $k a-\underline{d}$ ălika & 2 & 18 \\
\hline Insgesamt & 107 & 178 \\
\hline
\end{tabular}

Insgesamt wurden 285 einfache Verbalsätze, deren Subjekt „Gott“ ist, gesammelt. 178 Sätze besitzen die Wortstellung VS, 107 Sätze dagegen die Wortstellung SV.

Es gibt sieben Ursachen für die Voran- bzw. Nachstellung des Subjekts „Gott“ im Koran:

1. Tempus und Aspekt: Verben im Perfekt, die eine einmalige und abgeschlossene Handlung ausdrücken, stehen am Satzanfang. Zu dieser Gruppe gehören Verben wie qāla, ğa'ala, halaqa und Verben, die in erzählenden Abschnitten des Korans erscheinen bzw. Vordergrundinformation ausdrücken. Diese Sätze tragen die Hauptinformation und bringen die Ereignisse im Text voran. Außerdem folgt ihre Handlung zeitlich auf die Handlung der voraufgehenden Sätze, welche also gewissermaßen ihre Voraussetzung bilden.

Verben im Imperfekt, die eine zukünftige und einmalige Handlung ausdrücken, stehen am Satzanfang und folgen dem Subjekt. In diesen Sätzen geht es um eschatologische Schilderungen, die zukünftige Strafe und den Lohn der Gläubigen und der Ungläubigen.

Verben im generellen Präsens sind zeitstufenlos, d.h. ihr zeitlicher Skopus ist unbegrenzt und sie drücken eine wiederholte Handlung aus. Laut Bloch (1949) ist SV die 
häufigste Wortstellung in Sätzen der Poesie, die einen gnomischen Inhalt haben. Statistisch gesehen ist SV auch die übliche Wortstellung der Sätze im Koran, deren Verb im generellen Präsens steht. 85 Sätze besitzen die Wortstellung SV im Vergleich zu 35 Sätzen mit der Wortstellung VS. Dazu kommen 34 Sätze mit der Wortstellung VS, deren Verb zwar im Perfekt steht, aber die gleiche Funktion hat wie das generelle Präsens.

Eine große Anzahl der Sätze mit dem Subjekt „Gott“ enthalten ein Verb im gnomischen Präsens (154 Verben). Dies hängt damit zusammen, dass es in diesen Sätzen um die Macht Gottes geht: die Verben bezeichnen unbegrenzte und ewige Handlungen.

2. Die Funktionen der Partikel: Die Wortstellung nach sa-, sawfa, qad und la-qad ist VS, während die Wortstellung nach den Partikeln bal und tumma VS oder SV ist.

Wenn tumma als Verbindungspartikel zweier Sätze dient, die zeitlich aufeinanderfolgen, ist die Wortstellung VS.

Die Adversativpartikel bal verlangt die Wortstellung VS, während die Wortstellung SV erscheint, wenn bal als Betonungspartikel dient.

3. Auch die Wortstellung nach dem Bestimmungsadverb ka-dālika bezieht sich auf seine Funktion. Das anaphorische Pronomen verlangt die Wortstellung VS. In zwei Sätzen, deren Wortstellung SV ist, dient ka-dālika als das Subjekt des Satzes.

4. Wortstellung als rhetorisches bzw. stilistisches Mittel: Eines der wichtigsten Themen des Korans, welches sich wiederholt, ist die Macht Gottes und sein Verhältnis zu den Gläubigen und den Ungläubigen. Wiederholungen sind verbreitet im Koran, wie Nöldeke schreibt:

Daß der Korān seine Hauptsätze immer wiederholt, und zwar gern mit denselben Worten, macht ihn uns oft recht langweilig. Das hatte aber für Muhammed seine gute Berechtigung; ihm kam es gerade darauf an, seine Lehre durch solche Wiederholungen recht einzuschärfen. Und die Araber, die an feste Formeln gewöhnt waren, hat es gewiß nicht befremdet, wenn er an verschiedenen Stellen auch für Nebensachen dieselben oder doch wesentlich dieselben Formeln gebraucht. ${ }^{102}$

Trotz dieser Wiederholungen könnte es sein, dass Muhammad sich über die Notwendigkeit der Variation bewusst war, wobei neue oder nicht bekannte Ausdrücke immer die Aufmerksamkeit der Hörer auf sich ziehen.

Die unterschiedlichen Wortstellungen werden in zwei Fällen gebraucht, um Wiederholungen derselben Struktur zu vermeiden: In den Sätzen mit generellem Präsens (Kategorie 2) und in den Sätzen, die Gott als Schöpfer darstellen (Kategorie 1.2).

5. Nachdruck: In fünf Fällen steht das Subjekt „Gott“ betont am Satzanfang:

Kategorie 1.2: Die 13 SV-Sätze, deren Verb im Perfekt steht.

Kategorie 3: Sure 45:26

Kategorie 8: Sure 29:20

Kategorie 10: Sure 2:113 und Sure 4:141.

Kategorie 12: Sure 5:67

102 NÖLDEKE 1910/1982: 7.

$J A I S \cdot 14$ (2014): 63-86 
Was diese Sätze gemein haben, ist ihr paralleles Satzgefüge bestehend aus einem Subjekt, dem Relativpronomen alla $\underline{d} \bar{\imath}$ und Verb. Die Betonung ist dabei deutlich, denn die Sätze äußern, dass nur derjenige gut ist, der alles geschöpft hat und im Jenseits die Menschen verurteilen wird.

Isolierung wird auch in Sätzen „mit zwei Gesichtern“ durchgeführt, in denen das isolierte Demonstrativpronomen sich auf die vorher erwähnten Gläubigen bezieht. Durch diese Struktur wird betont, wer Gottes Lohn bekommt.

6. Sätze mit der Negativpartikel $l \bar{a}$ haben zwei Wortstellungstypen: VS und SV. Die zwei Wortstellungstypen lassen sich dadurch voneinander unterscheiden, dass in den SV-Sätzen die Verben im generellen Präsens stehen und sich wiederholen. Die VS-Sätze dagegen sind inhaltlich unterschiedlich und tragen die Hauptinformation des Verses. Es finden sich zwei weitere Sätze, deren Wortstellung SV ist und die durch die Partikel $m \bar{a}$ eingeleitet werden. Laut Ğurğān̄̄ weißt die Voranstellung des Subjekts in Negativsätzen darauf hin, dass nur die Identität der Täter, nicht aber die Handlung an sich negiert wird.

7. Das Subjekt „Gott“ in Fragesätzen unterliegt der zweiten Stellung, d.h. VS.

\section{Bibliographie}

ABDEl HaleEM, Mohammad. 2004. "Grammatical Shift for Rhetorical Purposes: Iltifāt and related features in the Qur'ān". In: Colin TuRner (ed.), The Koran: Critical Concepts in Islamic Studies, London and New York 2004: 159-193.

BELL, Richard. 1953. Introduction to the Qur'an. Edinburgh.

BLOCH, Alfred. 1946 Vers und Sprache im Altarabischen. Basel.

BobZIN, Hartmut. 2010. Der Koran. München.

CRYSTAL, David. 2003. A Dictionary of Linguistics and Phonetics (fifth edition). Oxford.

DAHLGREN, Sven-Olof. 2001. "Word Order and Topicality in the Qur'ān". Zeitschrift für arabische Linguistik, 39: 20-35.

EITAN, Israel. 1929. "Hebrew and Semitic particles: Comparative studies in Semitic philology". The American Journal of Semitic Languages and Literatures, 45: 48-63, 130-145, 197-211.

FISCHER, Wolfdietrich. 1972. Grammatik des klassischen Arabisch. Wiesbaden.

Givòn, Talmy. 1977. "The drift from VSO to SVO in Biblical Hebrew: the pragmatics of tense- aspect”. In: Charles N. Li (ed.), Mechanisms of Syntactic Change, London 1977: 182-254.

GIvòn, Talmy. 1984. Syntax: A Functional-Typological Introduction. Amsterdam and Philadelphia.

HOPPER, Paul J. 1979. "Aspect and foregrounding in discourse”. Discourse and Syntax 1979: 213241.

KHOURY, A. Theodor A. 2004. Der Koran. Arabisch-deutsche Übersetzung und wissenschaftlicher Kommentar. Gütersloh.

MCAulifFE, Jane D. (General Editor). 2002. Encyclopedia of the Qur'ān. Leiden-Boston.

MeHREn, August F. 1853. Die Rhetorik der Araber. Wien.

MurAOKA, Takamitsu. 1985. Emphatic Words and Structures in Biblical Hebrew. Jerusalem.

Mustansir, Mir. 2004. "Dialogue in the Qur'an”. In: Colin TuRnER (ed.), The Koran: Critical Concepts in Islamic Studies, London and New York 2004: 194-213.

MYHILL, John. 1992. "Word order and temporal sequencing". In: Doris 1. PAYNE (ed.), Pragmatics of Word Order Flexibility, Amsterdam/Philadelphia 1992: 266-278. 
NÖLDEKE, Theodor, „Zur Sprache des Korāns“, in: Beiträge und neue Beiträge zur semitischen Sprachwissenschaft 1982, 2-30. Strassburg 1910 (= repr. 1982)

PARET, Rudi. 1966. Der Koran (Übersetzung). Stuttgart.

PASHOVA, T. 2003. "The SV/VS alternation in modern written Arabic from a textual perspective". Zeitschrift für arabische Linguistik, 42: 7-40.

RECKENDORF, Herrmann. 1898. Die syntaktischen Verhältnisse des Arabischen. Leiden.

RECKENDORF, Herrmann. 1921. Arabische Syntax. Heidelberg.

ReusCHEL, Wolfgang 1969. Aspekt und Tempus in der Sprache des Korans. Habilitation Univ. Leipzig.

SuleimAn, Saleh M. 1989. "On the pragmatics of subject-object preposing in Standard Arabic". Language Science, 11: 215-235.

TuR-SinAI, Naftali H. 1945-1958. Die Heilige Schrift (neu ins Deutsche übertragen). Jerusalem.

$$
\begin{aligned}
& \text { ANīs, Ibrāhīm } \\
& \text { al-ZAMAHŠARI }
\end{aligned}
$$

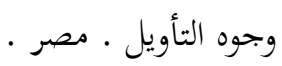

$$
\begin{aligned}
& \text { al-ĞURǦĀNī } \\
& \text { al-SUYŪṬT̄ }
\end{aligned}
$$

YEHUDIT DROR, University of Haifa, Department of Arabic Language and Literature, Israel $\varangle$ judror@gmail.com 